Trauma Berufskrankh 2016 · [Suppl 1]: 18:S18-S25 DOI 10.1007/s10039-015-0050-4

Online publiziert: 1. Juli 2015

(c) Springer-Verlag Berlin Heidelberg 2015

CrossMark

Frederic Welsch ${ }^{1} \cdot$ T. Stein ${ }^{1}$ P. Minzlaff ${ }^{1}$ R. Hoffmann ${ }^{2}$

${ }^{1}$ Abteilung Sportorthopädie, Knie- und Schulterchirurgie, Berufsgenossenschaftliche

Unfallklinik Frankfurt am Main, Frankfurt am Main, Deutschland

${ }^{2}$ Abteilung Unfallchirurgie und Orthopädische Chirurgie, Berufsgenossenschaftliche

Unfallklinik Frankfurt am Main, Frankfurt am Main, Deutschland

\title{
Kniegelenktrauma mit Knorpelabscherverletzung
}

\section{Refixation, Resektion, Sekundärversorgung?}

Chondrale bzw. osteochondrale Läsionen sind typische Verletzungsmuster in der Orthopädie und Unfallchirurgie, insbesondere bei Sportlern häufig in Kombination mit ligamentären Begleitverletzungen. Die Therapie stellt den Behandler vor eine große Herausforderung, da Knorpelgewebe nur eine geringe Selbstheilungspotenz aufweist und der Knorpelschaden unbehandelt zur vorzeitigen Arthrose führen kann [3]. Das Ziel sollte die Wiederherstellung eines stabilen, belastbaren und dauerhaft haltbaren Knorpels sein. Hyaliner Gelenkknorpel enthält weder Blutgefäße noch eine nervale Versorgung. Die Knorpelzellen werden über Diffusion aus der Synovia und v. a. vor Wachstumsende aus dem subchondralen Knochen ernährt. Aus diesen Gründen regeneriert insbesondere adulter Gelenkknorpel nach Verletzungen meist nur unvollständig. Die akute traumatische Knorpelabscherverletzung ist eine Sonderform der Knorpelläsion und hinsichtlich Diagnostik und Therapie von den degenerativen Knorpelveränderungen zu unterscheiden.

\section{Inzidenz}

Die traumatische osteochondrale Läsion kann alle Altersgruppen betreffen, die höchste Inzidenz liegt im Alter von ca. 20 Jahren [9].

Häufige Ursachen von traumatischen osteochondralen Verletzungen sind Patellaluxationen, bei denen es in bis zu $50 \%$ der Fälle zu Abscherungen des Knorpels an der medialen Patella, am First und an der lateralen Trochleakante kommen kann [20]. Auch bei Kreuzbandverletzungen entstehen im Rahmen des Rotationstraumas hohe Scherkräfte mit Knorpelverletzungen in 16-46\% der Fälle insbesondere am lateralen Femurcondylus $[2,15]$.

\section{Diagnostik}

Die Schwellung und der Schmerz sind die klinisch führenden Symptome, ggf. auch Blockaden. Die Punktion kann neben der therapeutischen Entlastung auch diagnostische Hinweise auf eine osteochondrale Verletzung geben, wenn im blutigen Punktat Fettaugen vorliegen. Zur Bildgebung gehört die Röntgenstandardaufnahme in 3 Ebenen. Größere osteochondrale Fragmente können häufig bereits hierdurch detektiert werden, wohingegen kleine Fragmente und insbesondere rein chondrale Fragmente der nativradiologischen Diagnostik meist entgehen. Die Magnetresonanztomographie (MRT) ist die sensitivste Untersuchungsmethode für den Knorpel. Hierzu ist insbesondere das hochauflösende 3-Tesla-MRT in der Gradientenechotechnik geeignet. Die höchste Sensitivität haben Protonendichte(PD)oder $\mathrm{PD} / \mathrm{T} 2$-gewichtete fettunterdrückte Turbospinecho(TSE)-Sequenzen [17]. Auch können durch die MRT therapierelevante Begleitverletzungen dargestellt werden. Kleine osteochondrale Fragmente sind im MRT manchmal nicht von Weichteilformationen zu unterscheiden. Um sie zu detektieren, kann hier ergänzend eine Computertomographie (CT) sinnvoll sein. Auch um sich einen Über- blick über die genaue Defektgröße, Morphologie und Lokalisation zu verschaffen, kann die CT eingesetzt werden. Letztendlich können aber nur durch die Arthroskopie die Beschaffenheit und die Integrität des Fragments exakt beurteilt werden [18].

\section{Verletzungsformen}

Bei der osteochondralen Verletzung kann sich das Fragment zum einen stabil oder instabil in situ befinden, zum anderen kann es vollständig abgelöst sein. Unterschieden werden rein chondrale von den häufiger auftretenden osteochondralen Fragmenten, denen noch eine Knochenlamelle anhängt. Das Fragment selbst kann intakt oder in sich frakturiert sein [12].

\section{Therapiestrategien}

Evidenzbasierte Therapieempfehlungen hinsichtlich der Versorgung osteochondraler Verletzungen am Kniegelenk gibt es nicht. Kühle et al. [9] veröffentlichte 2013 eine Metaanalyse zur Therapie der osteochondralen Verletzung am Kniegelenk, in die 19 Studien mit insgesamt 638 Patienten eingeschlossen wurden. Diese waren alle Studien mit Evidenzlevel IV, teilweise nur Fallserien. Dementsprechend ergab sich im Coleman-Methodik-Score, einem Score zur Analyse der Studienwertigkeit, ein sehr niedriger Wert von 29 Punkten (von 100 möglichen). Das Fazit war, dass basierend auf der vorliegenden wissenschaftlichen Datenlage keine eindeutigen Handlungsempfehlungen hinsicht- 


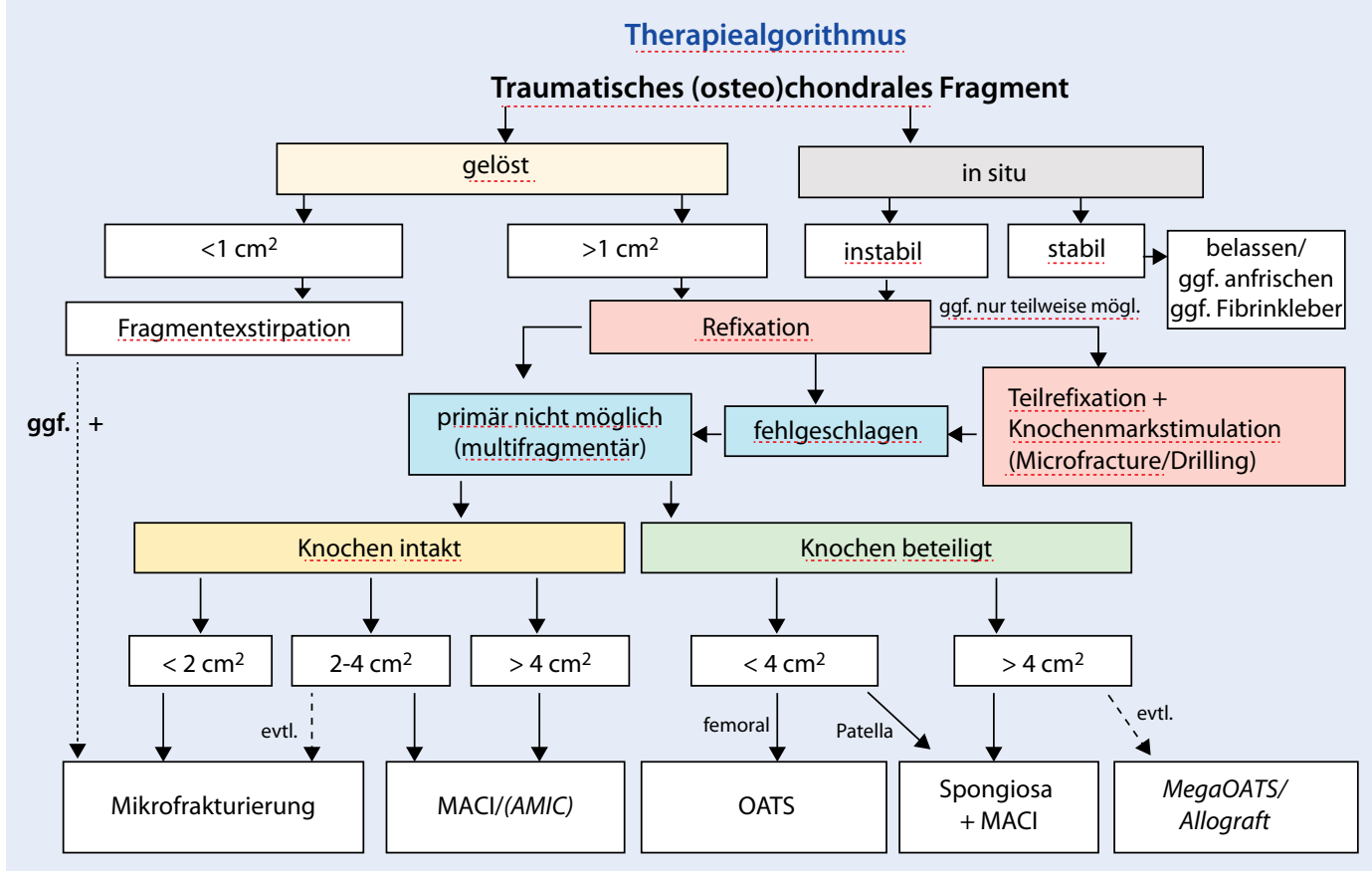

Abb. $1<$ Behandlungsalgorithmus. AMIC autologe matrixinduzierte Chondrogenese, $\mathrm{MACl}$ matrixgestützte autologe Chondrozytenimplantation, OAT osteochondraler autologer Transfer
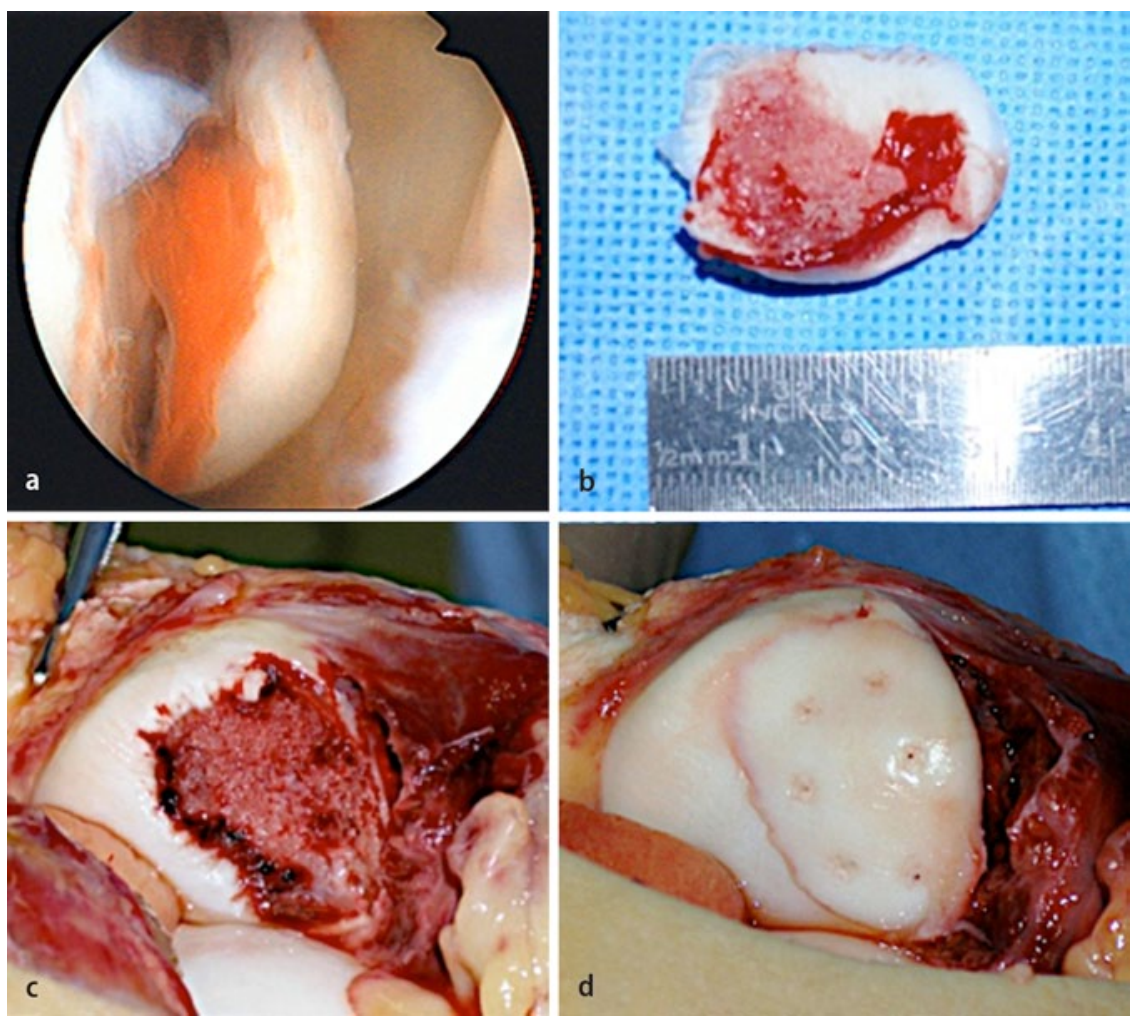

Abb. $2 \Delta$ Patellaluxation mit osteochondralem Fragment. a Fragment im lateralen Recessus, b Knorpelknochenfragment, $\mathbf{c}$ Defekt an Patella, $\mathbf{d}$ Refixationsergebnis mit PLLA-Pins

lich dieser Verletzung ausgesprochen werden können. Offene Fragen bezüglich der Therapie osteochondraler Verletzungen bleiben:
- Welche Fixationsmaterialen (Metall/ bioresorbierbar/Fibrinkleber) sollten verwendet werden?

- Was ist die kritische Fragmentgröße?
- Welche Rolle spielt das Intervall Unfall zu Versorgung?

- Wie sollen rein chondrale Verletzungen therapiert werden?

- Gibt es prognostische Parameter (Patientenalter, Begleitverletzung, Defektlokalisation)?

- Sind regenerative Knorpeltherapien der Refixation oder Fragmentexstirpation überlegen?

- Wie sind die Langzeitfolgen dieser Verletzung?

\section{Aspekte der Therapie}

Es besteht allgemeiner Konsens, dass eine Refixation zu bevorzugen ist.

\section{Fixationstechnik}

Hinsichtlich der Fixationstechniken werden metallische Implantate (Schrauben/ Drähte), Fibrinkleber sowie bioresorbierbare Pins und Schrauben verwendet [14].

Vorteil der Metallschrauben ist die stabile Fixation mit gutem Kompressionseffekt. Nachteile sind das unterschiedliche Elastizitätsmodul zum Knorpel, die notwendige Perforation des Knorpelfragments und ggf. die Schädigung des korrespondierenden Knorpels sowie die eingeschränkte MRT-Beurteilbarkeit und der meist notwendige Zweiteingriff zur Ent- 
fernung [27]. Implantate aus Titan sind dabei zu bevorzugen.

Die alleinige Verwendung von Fibrinkleber hat den Nachteil einer geringen Primärstabilität mit der Erfordernis einer immobilisierenden Nachbehandlung [7]. Fibrinkleber birgt ein Restrisiko für Krankheitsübertragung durch seinen Serumbestandteil Fibrinogen [19]. Vorteile der Fibrinkleberverwendung sind, dass der Knorpel nicht perforiert wird, die Bildung von Gap-Junctions reduziert wird und eine arthroskopische Applikation möglich ist [19]. Fibrinkleber sollte nur bei kleinen Fragmenten oder additiv $\mathrm{zu}$ anderen Fixationstechniken eingesetzt werden [14].

Der Trend geht zur bioresorbierbaren Fixation. Sie reduziert das Risiko der Knorpelschädigung und erfordert keinen Zweiteingriff zur Entfernung [28]. Allerdings ist die mechanische Stabilität im Vergleich zu den metallischen Implantaten reduziert und es können durch die Resorption entzündliche Gewebereaktionen am Knochen, z. B. Zysten [5], oder an der Synovialis v. a. bei Implantaten mit Polyglykolsäureanteil auftreten [1]. Die Gewebeverträglichkeit ist bei bioresorbierbaren Implantaten aus Poly-L-Laktidsäure (PLLA) am besten mit vergleichbarer Biokompatibilität zu metallischen Implantaten [13]. Durch die Entwicklung der sog. Self-Reinforcement-Eigenschaften, d. h. bioresorbierbarer Implantate mit funktionsorientierter Molekularausrichtung, konnte auch deren mechanische Stabilität verbessert werden [21].

\section{Zeitpunkt der operativen Versorgung}

In einer In-vitro-Studie wurde gezeigt, dass es innerhalb von wenigen Stunden nach Fragmentisolation zu einem signifikanten Anschwellen des Fragments auf bis zu $50 \%$ des Knorpelvolumens kommt [16].

In einer weiteren Tiermodellstudie konnte nachgewiesen werden, dass die Zeitdauer der Fragmentisolation negativ mit der Knorpelqualität korreliert, d. h. die Expression von extrazellulärer Matrix und Kollagen Typ II sinkt. Auch die Knorpeldegeneration nach erfolgter Refixation zeigte eine Abhängigkeit von der

Trauma Berufskrankh 2016 • [Suppl 1]: 18:S18-S25 DOI 10.1007/s10039-015-0050-4

(c) Springer-Verlag Berlin Heidelberg 2015

F. Welsch · T. Stein · P. Minzlaff · R. Hoffmann

Kniegelenktrauma mit Knorpelabscherverletzung. Refixation, Resektion, Sekundärversorgung?

\section{Zusammenfassung}

Hintergrund. Obwohl osteochondrale Verletzungen am Knie häufig bei Patellaluxationen und Bandverletzungen auftreten, existieren in der Literatur keine evidenzbasierten Therapiestrategien. Unbehandelt können sie zu Arthrose führen. Ziel muss daher die Wiederherstellung eines dauerhaft belastbaren hyalinen Knorpels sein. Vorgestellt wird der eigene Behandlungsalgorithmus.

Therapie. Es besteht allgemeiner Konsens, traumatisch abgelöste Knorpelfragmente, sofern geeignet, frühzeitig zu refixieren. Kleinere Fragmente $<1 \mathrm{~cm}$ können entfernt werden, der Defekt in der Belastungszone lässt sich mit knochenmarkstimulierender Therapie behandeln. Größere Fragmente sollten refixiert werden. Hierfür stehen bioresorbierbare Implantate zur Verfügung, die gegenüber den Metallimplantaten mittlerweile eine ähnlich gute Stabilität und Biokompatibilität aufweisen. Vorteile sind die Reduktion von korre- spondierenden Knorpelschäden und die Vermeidung des Zweiteingriffs zur Implantatentfernung. Auch rein chondrale größere Fragmente haben eine Einheilungspotenz und sollten als First-Line-Therapie insbesondere bei jüngeren Patienten refixiert werden. Bei nichtrefixierbaren Fragmenten oder Versagen der Refixation kommen in Abhängigkeit von der Größe, Lokalisation und Knochenbeteiligung die knorpelreparativen Verfahren wie Mikrofrakturierung, osteochondraler Transfer oder zellbasierte Verfahren zur Anwendung. Schlussfolgerung. Die (osteo)chondrale Refixation zeigt gute klinische Ergebnisse und sollte wenn immer möglich primär frühzeitig angestrebt werden.

Schlüsselwörter

Knieverletzung $\cdot$ Kniegelenk $\cdot$ Knorpel .

Patellaluxation · Frakturfixation

\section{Traumatic knee injury with osteochondral lesions. Refixation, resection, secondary treatment?}

\section{Abstract}

Background. Although osteochondral lesions of the knee are frequently associated with patellar dislocation and ligament injuries, there are no evidence-based strategies for therapy. Without treatment they can lead to osteoarthritis; therefore, the aim is to restore a durable and resilient cartilage. This article presents an in-house treatment algorithm.

Therapy. There is general agreement that detached cartilage fragments should be refixed early, if suitable. Small fragments less than $1 \mathrm{~cm}$ in size can be removed and a defect in the load-bearing zone should be treated with bone marrow stimulation techniques. Major fragments should be refixed. For this purpose bioresorbable implants are available, which now have a similar stability and biocompatibility to metallic implants with the advantages of reducing corresponding cartilage lesions and avoiding removal. Even major pure chondral fragments have the potential for healing and should be refixed as the first line treatment, especially in younger patients. If a fragment is not amenable for refixation or in cases of treatment failure, cartilage restorative and reparative procedures, such as microfracturing, osteochondral transfer or chondrocyte transplantation are used depending on the size, location and bone involvement.

Conclusion. Osteochondral refixation shows good clinical results and should be primarily pursued whenever possible.

\section{Keywords}

Knee injuries · Knee joint · Cartilage · Patellar dislocation · Fracture fixation
Zeitdauer der vorangegangenen Isolation. Nach Refixation war aber auch teilweise eine Regeneration des Knorpels zu beobachten [22]. In einem Fallbericht wurde die erfolgreiche Refixation eines osteochondralen Fragments der Patella 8 Wochen nach Trauma dokumentiert [6].
Die Refixation sollte also so zeitnah wie möglich erfolgen, aber auch ältere Fragmente haben durchaus eine Refixations- und Regenerationschance $[4,6,22]$. 

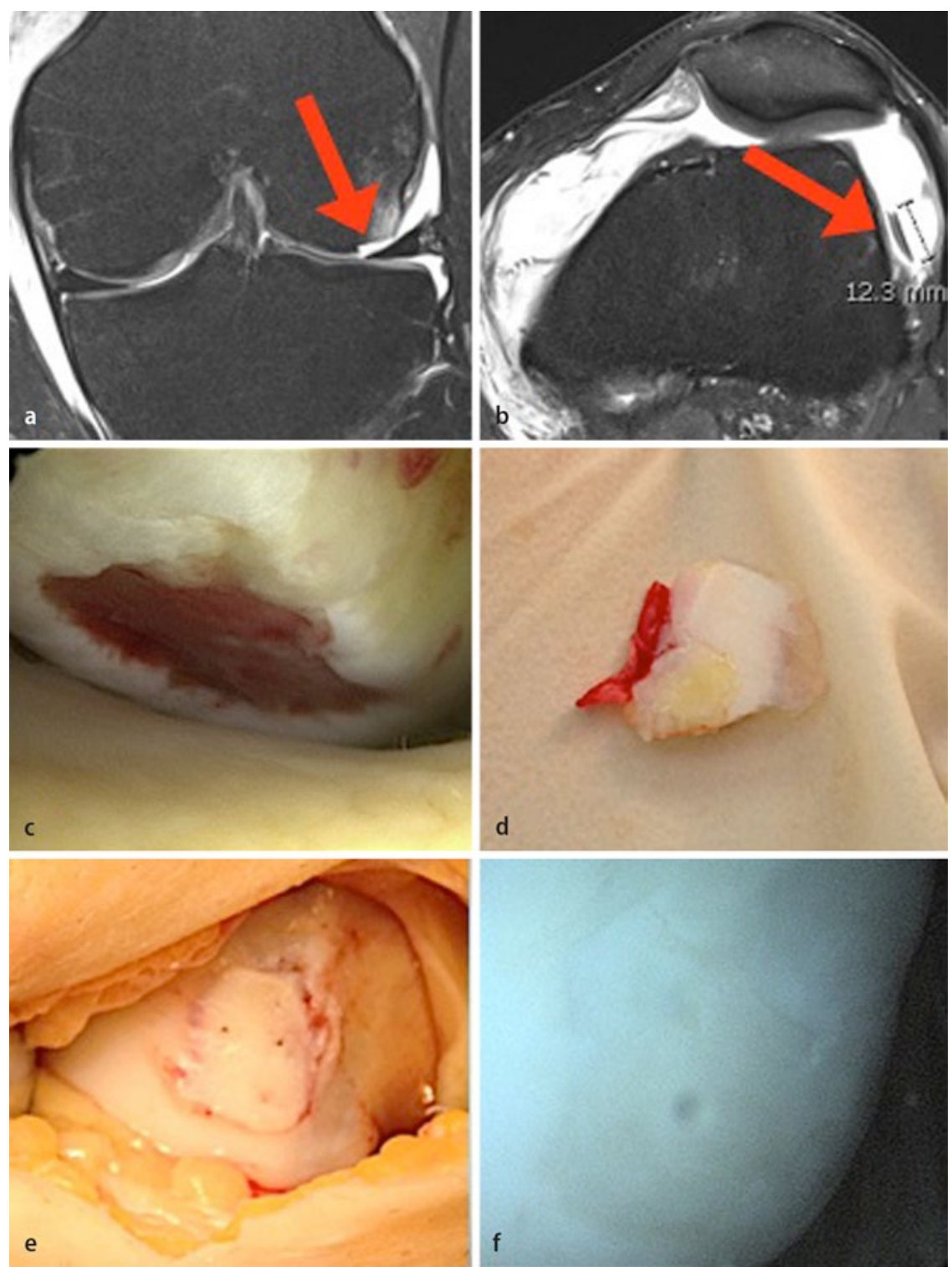

d

f

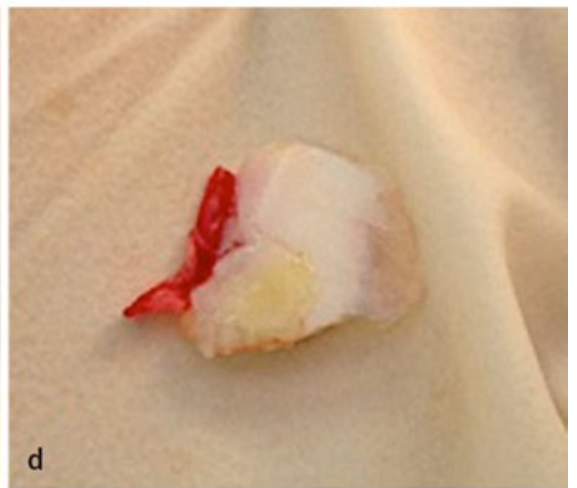

Abb. $3 \Delta$ Patellaluxation mit chondralem Fragment vom lateralen Femurcondylus. a,b Magnetresonanztomographie, $\mathbf{c}$ arthroskopischer Befund lateraler Femurcondylus, $\mathbf{d}$ chondrales Fragment, e Defekt Femurcondylus, f Refixationsergebnis mit PLLA-Pins ,second look“ 5 Monate postoperativ

\section{Eigenschaften der Knorpelfragmente}

Bei rein chondralen Läsionen, d. h. ohne anhängenden Knochen am Fragment, ist die Datenlage sehr spärlich und es wird diskutiert, ob eine Refixation überhaupt sinnvoll erscheint. In der Studie mit der größten Fallzahl nach osteochondraler Refixation wurden auch die Refixationsergebnisse rein chondraler Fragmente nachuntersucht. Hierbei waren nur 50\% der Refixationen rein chondraler Fragmente erfolgreich, wobei diese nur mit Fibrinkleber erfolgten. Die Refixationser- gebnisse waren bei makroskopisch intakten chondralen Fragmenten besser verglichen mit traumatisierten oder frakturierten Fragmenten. Die günstigste Prognose hatten osteochondrale oder chondrale intakte Fragmente [12]. Ein Fallbericht beschreibt die erfolgreiche Refixation mit Ethipin ${ }^{\circ}$ eines rein chondralen frischen Fragments bei einem 11-jährigen Jungen im Bereich der Trochlea. In der SecondLook-Arthroskopie und Histologieuntersuchung konnten eine komplette Fragmentheilung und ein normaler Knorpel nachgewiesen werden [11]. In einem aktuelleren Fallbericht wurde bei 3 jugend- lichen Patienten ein rein chondrales Fragment mit bioresorbierbaren Pins refixiert. Zwei Jahre postoperativ zeigte sich klinisch und MR-tomographisch eine erfolgreiche Einheilung [23]. In einer weiteren Studie mit überwiegend rein chondralen Läsionen waren die Ergebnisse in den klinischen Scores gut. Im MRT zeigte sich kein „Full-Thickness-Defekt“, aber in 6 von 8 Fällen war die Knorpeldicke reduziert [26].

Wir erachten daher auch bei rein chondralen Läsionen einen Refixationsversuch als First-Line-Therapie als gerechtfertigt, insbesondere bei größeren Defekten und bei jüngeren Patienten.

\section{Therapiealgorithmus}

Im Falle einer noch in situ befindlichen stabilen Knorpelabscherläsion kann diese belassen und ggf. angefrischt bzw. mit Fibrinkleber additiv fixiert werden (• Abb. 1). Ist diese Abscherläsion instabil, sollte sie mit auflösbaren Pins in Abhängigkeit von der Lokalisation arthroskopisch oder offen fixiert werden. Falls nur eine Teilrefixation möglich ist, kann ergänzend eine knochenmarkstimulierende Therapie (z. B. Mikrofrakturierung) erfolgen. Bei komplett gelöster (osteo)chondraler Läsion ist die Therapie abhängig von der Größe und Knorpelqualität (multifragmentär). Im Falle eines gelösten Fragments unter $1 \mathrm{~cm}$ Durchmesser kann dieses arthroskopisch entfernt werden. Falls sich im Defektbett Blut („Primärclot") befindet, sollte dies belassen werden, um die Möglichkeit zur Bildung eines Knorpelregenerats zu erhalten. Liegt der Knochen frei, wird eine knochenmarkstimulierende Therapie im Defektbett durchgeführt.

Bei über $1 \mathrm{~cm}$ großen chondralen oder osteochondralen Fragmenten sollte eine Refixation angestrebt werden [18]. Wir führen dies in Abhängigkeit der Fragmentgröße bei kleineren oder rein chondralen Fragmenten mit "chondral darts“ (PLLA-Pins, - Abb. 2, 3) oder bei größeren Fragmenten mit Biokompressionsschrauben aus PLLA durch. Selbst bei mehrfragmentären Fragmenten kann ein Refixationsversuch erfolgreich sein (• Abb. 4). Gegebenenfalls können auch nur eine Teilrefixation des noch qualitativ 

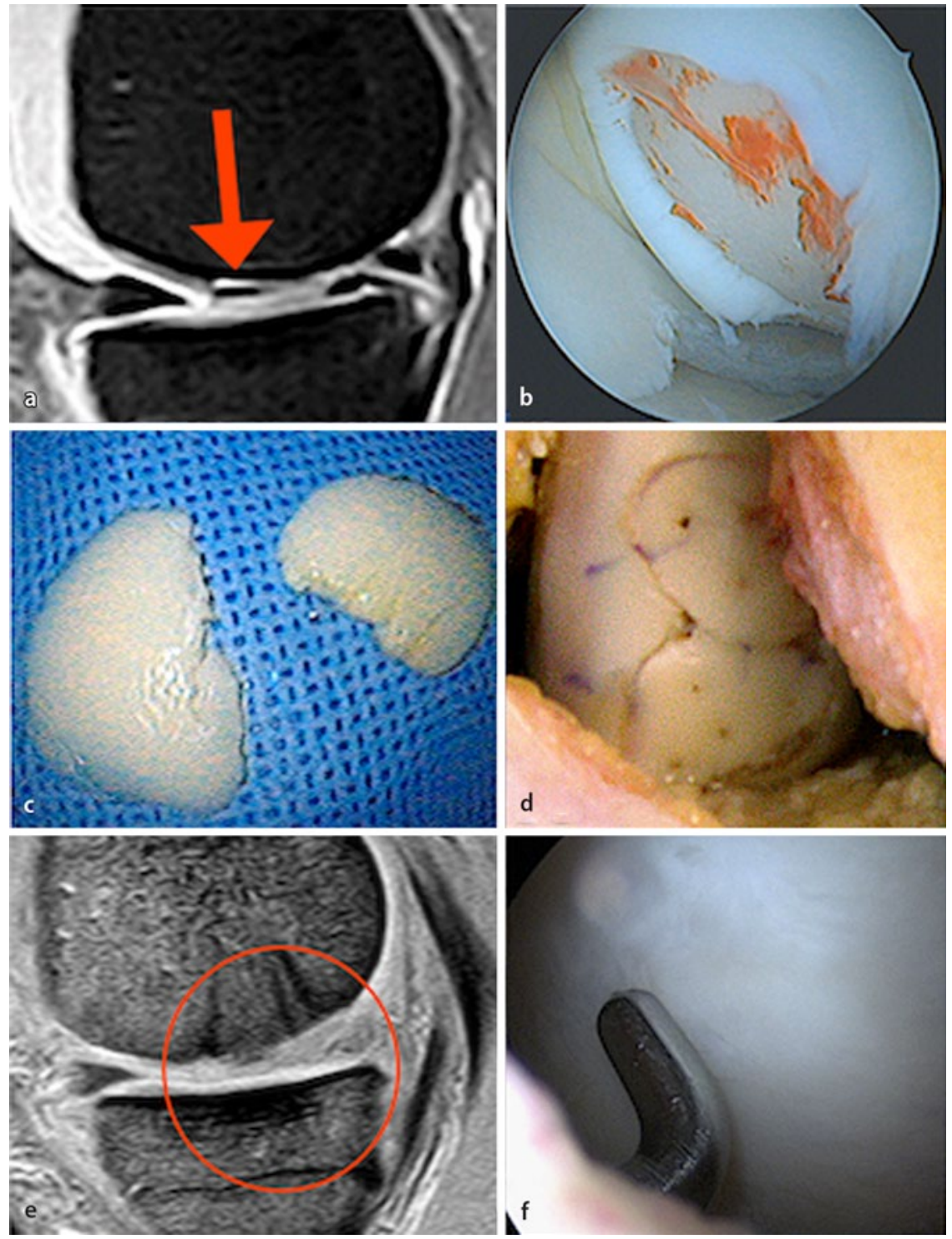

Abb. 4 A Mehrfragmentäre chondrale Läsion vom medialen Femurcondylus. a Magnetresonanztomographie (MRT), b Defekt Femurcondylus arthroskopisch, c Knorpelfragmente, $\mathbf{d}$ Refixation mit

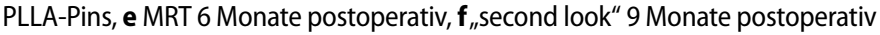

guten Fragments und eine Knochenmarkstimulation des verbliebenen Defektbetts erfolgen.

Ist eine primäre Refixation des Fragments nicht möglich bzw. die Refixation fehlgeschlagen, kommen die knorpelreparativen Techniken zum Tragen. Die Art dieser Therapie ist abhängig von der Beschaffenheit des subchondralen Knochens, der jeweiligen Größe und der Lokalisation des Defekts. Ist der subchondrale Knochen intakt und die Defektgröße gering $\left(<2 \mathrm{~cm}^{2}\right)$, kann eine knochenmarkstimulierende Technik angewandt werden. Bei Defekten zwischen 2 und $4 \mathrm{~cm}^{2}$ ist in Abhängigkeit von Patienten- alter, Anspruch und Lokalisation (Belastungszone) eine alleinige knochenmarkstimulierende Technik, besser jedoch in Kombination mit einer Matriximplantation (autologe matrixinduzierte Chondrogenese, AMIC), oder ein zellbasiertes Verfahren (matrixgestützte autologe Chondrozytenimplantation, MACI) indiziert. Letzteres wird bei Defekten über $4 \mathrm{~cm}^{2}$ angewandt (- Abb. 5).

Ist der subchondrale Knochen mit beteiligt, hat sich bei Defekten unter $4 \mathrm{~cm}^{2}$ der Knorpel-Knochen-Transfer (OAT) an Femur und Trochlea bewährt. An den anderen Lokalisationen und bei Defekten über $4 \mathrm{~cm}^{2}$ sollte eine Spongiosaplas- tik in Kombination mit einer MACI gewählt werden. Alternativen hierzu wären Allografts oder die Mega-OATS-Technik in Abhängigkeit von den logistischen Voraussetzungen und der Erfahrung des Behandlers mit diesen Techniken.

Allgemein gilt für die Therapie von osteochondralen Verletzungen, dass Begleitpathologien wie Achsfehlstellungen, Instabilitäten (Patella, Kreuzbänder) für den langfristigen Erfolg der Knorpeltherapie mit behandelt werden sollten [24]. Ob dies einzeitig oder im Verlauf durchzuführen ist, wird noch diskutiert und sollte im Einzelfall entschieden werden [4].

\section{Nachbehandlung}

Die Nachbehandlung sollte immer individualisiert abhängig von Operationstechnik, Defektlokalisation, Defektgröße, Begleitpathologien und angepasst an den Rehabilitationsverlauf (Muskelstatus, Beweglichkeit, Koordination) erfolgen. Wir verwenden postoperativ eine passive Bewegungsschiene (CPM) für 4 bis 6 Wochen. Randomisierte kontrollierte HighLevel-Studien bezüglich der Effizienz der CPM-Therapie liegen zwar bis dato nicht vor, allerdings zeigt sich in tierexperimentellen Studien, dass es über die Stimulation der zellulären Antwort zu einer signifikanten Verbesserung der Beweglichkeit und der histologischen, biochemischen und biomechanischen Eigenschaften des Knorpels kommt [8].

Auch die Rückkehr zum Sport muss individuell abhängig vom Operationsverfahren und von den muskulären und propriozeptiven Fähigkeiten gestaltet werden. Diesbezügliche Daten für osteochondrale Refixationen existieren in der Literatur nicht. Allerdings kann die Entscheidung in Anlehnung an die knorpelreparativen Therapien erfolgen: bei OAT nach 6 Monaten, bei Mikrofrakturierung nach 8 bis12 Monaten, bei zellbasiertem Verfahren nach 18 bis 25 Monaten [10].

\section{Ergebnisse}

In der Studie mit den größten Fallzahlen hinsichtlich der Refixation chondraler und osteochondraler Läsionen mittels resorbierbaren Pins und Fibrinkleber zeigte sich nach einer mittleren Nach- 

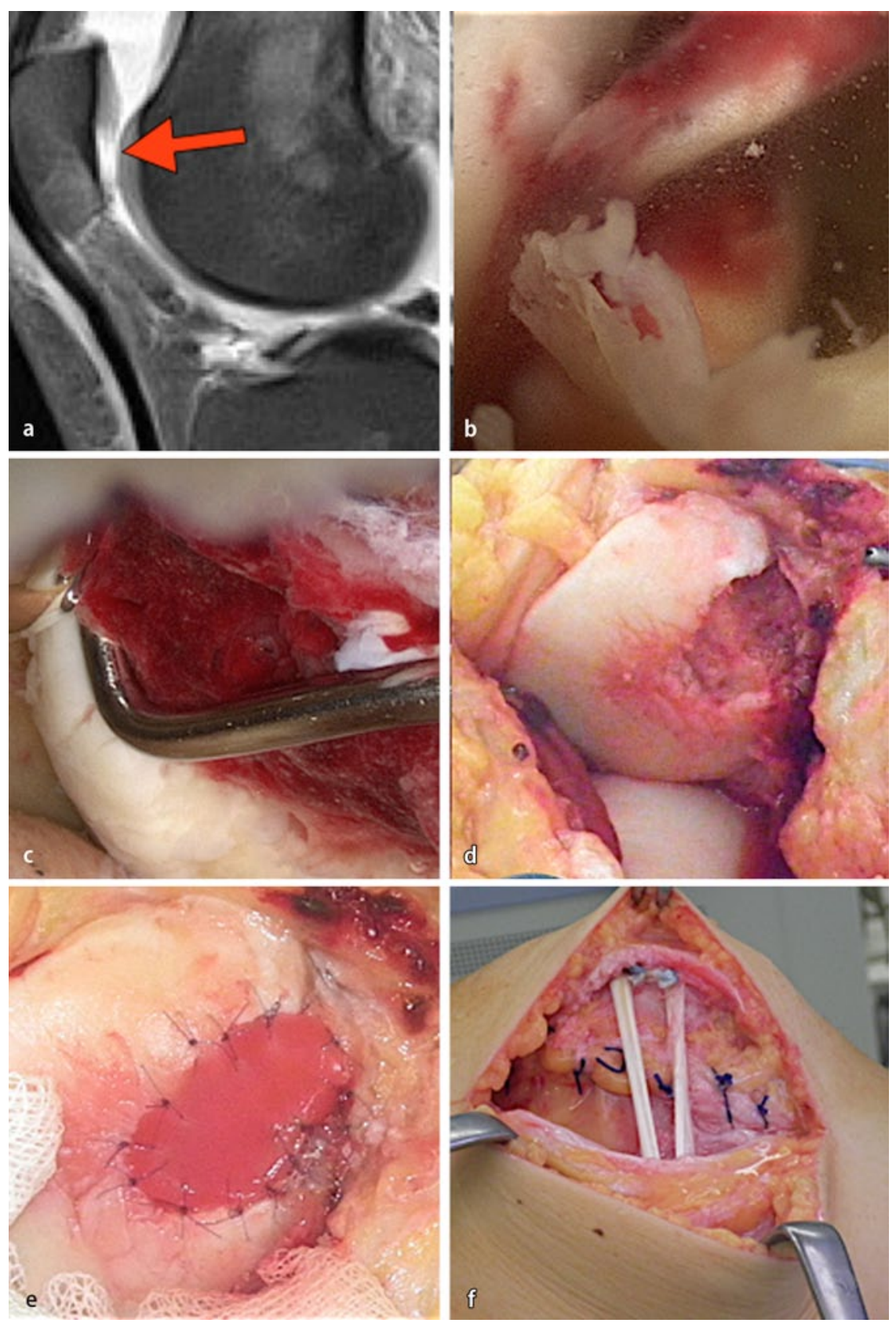

Abb. 5 Patellaluxation mit nichtrefxierbarem Fragment mediale Patellafacette. a Magnetresonanztomographie, $\mathbf{b}$ mehrfragmentäres Fragment, $\mathbf{c}$ Patelladefekt arthroskopisch, $\mathbf{d}$ Patelladefekt offen, $\mathbf{e}$ matrixgestützte autologe Chondrozytenimplantation (MACl), f gleichzeitige Rekonstruktion mediales patellofemorales Ligament mit freier Gracilissehne

untersuchungszeit im MRT oder einer Second-Look-Arthroskopie bei den osteochondralen Läsionen eine Erfolgsrate von $95 \%$ mit keinen oder nur geringgradigen Knorpelschäden ([12], • Tab. 1). Ungünstiger waren die Ergebnisse bei den rein chondralen Läsionen. Hier betrug die Erfolgsrate nur $50 \%$. Revisionen waren überwiegend nur bei den chondralen und fragmentierten Läsionen erforderlich [12].
Neuere Studien mit kleinen Fallzahlen bei jugendlichen Patienten mit rein chondralen Läsionen, die mittels bioresorbierbarer Pins fixiert wurden, wiesen jedoch sehr gute klinische Ergebnisse und eine gute Einheilungsrate auch für diese Verletzungsform auf $[11,23,26]$. In einer aktuelleren Studie zu mittelfristigen Ergebnissen nach osteochondraler Refixation mit resorbierbaren Implantaten zeigten sich im mittleren Follow-up von 6,5 Jahren sehr gute klinische Ergebnisse mit einem Lysholm-Score von 89 Punkten [25]. Die guten klinischen Ergebnisse konnten in einer weiteren Multicenterstudie bestätigt werden [4]. In mehreren Studien ließ sich MR-tomographisch eine gute Integration des refixierten Knorpelfragments erkennen, jedoch waren bei einem Teil der Patienten eine Reduktion der Knorpelhöhe und teilweise kleinzystische Veränderungen nachzuweisen [12, 25, 26]. Allgemein zeigte sich in den Studien keine Korrelation zwischen den guten klinischen Ergebnissen und den teilweise bestehenden Veränderungen im MRT $[25,26]$.

\section{Fazit für die Praxis}

- Zur Therapie osteochondraler Verletzungen existieren keine Studien mit hohem Evidenzlevel.

- Eine frühzeitige operative Versorgung ist anzustreben, um eine Knorpelschädigung und das Aufquellen zu reduzieren.

- Kleine instabile Fragmente $<1 \mathrm{~cm}$ können reseziert werden, ggf. in Kombination mit einer knochenmarkstimulierenden Technik.

- Fragmente $>1 \mathrm{~cm}$ in der Belastungszone sollten refixiert werden. Intakte osteochondrale Fragmente weisen dabei bessere Heilungsergebnisse auf als multifragmentierte oder rein chondrale Fragmente. Trotzdem sollte auch bei größeren rein chondralen Fragmenten ein Refixationsversuch unternommen werden, insbesondere bei jüngeren Patienten.

- Bei primär nichtrefixierbaren Fragmenten oder nach gescheiterter Refixation kommen in Abhängigkeit von Lokalisation, Größe und Beteiligung des Knochens die verschiedenen knorpelreparativen Verfahren zur Anwendung.

- Begleitpathologien sollten beachtet und mit behandelt werden. 


\begin{tabular}{|c|c|c|c|c|c|c|c|}
\hline Autor & LOE & Patientenzahl & Verletzungstyp & Versorgung & FU (Jahre) & Ergebnis & Schlussfolgerung \\
\hline $\begin{array}{l}\text { Paar et al. } \\
{[12]}\end{array}$ & IV & $\begin{array}{l}118 \\
32\end{array}$ & $\begin{array}{l}\text { osteochondral } \\
\text { chondral }\end{array}$ & $\begin{array}{l}\text { Fibinkleber } \\
(14 x+\text { Ethipin }) \\
\text { Fibrinkleber }\end{array}$ & $\begin{array}{c}2,5 \\
\text { MR } \\
\text { teilweise second look-ASK }\end{array}$ & $\begin{array}{l}95 \% \text { Erfolgsrate } \\
50 \% \text { Erfolgsrate }\end{array}$ & $\begin{array}{l}\text { günstigste Prognose osteochondrales } \\
\text { oder intaktes chondrales Fragment }\end{array}$ \\
\hline $\begin{array}{l}\text { Chotel et al. } \\
\text { [4] }\end{array}$ & IV & $\begin{array}{c}14 \\
(11-15 J)\end{array}$ & osteochondral & $\begin{array}{l}5 \times \text { Schrauben } \\
4 \times \text { Biopin } \\
5 \times \text { Auszugsnaht } \\
\text { ggf. + Fibrinkleber }\end{array}$ & $2,5(15-89)$ & $\begin{array}{l}\text { keine Revision } \\
\text { IKDC 2000 } \\
\text { sehr zufrieden } \\
\text { zufrieden }\end{array}$ & $\begin{array}{l}\text { Erfolgreiche Refixation chondrale/ } \\
\text { osteochondrale Fragmente bei Kindern } \\
\text { auch } 2 \text { Monate nach Unfall möglich }\end{array}$ \\
\hline $\begin{array}{l}\text { Wachowski et } \\
\text { al.[25] }\end{array}$ & IV & $\begin{array}{c}12 \\
(14-39 \mathrm{~J}) \\
8 \text { Knie }-4 \text { Talus }\end{array}$ & osteochondral & \begin{tabular}{|l} 
bioresorbierbar PLLA \\
(Pin/Nail/Schraube)
\end{tabular} & $\begin{array}{l}6,5(5-7,9) \\
\text { MR }\end{array}$ & \begin{tabular}{|lr} 
Knie: & \\
Lysholm & 88,9 \\
Mc Dermott & 94,1 \\
Henderson Score (MR): & 11,6
\end{tabular} & $\begin{array}{l}\text { - gute klinische Ergebnisse } \\
\text { - MRT zeigt Veränderungen } \\
\text { - keine Korrelation Klinik - MRT } \\
\text { - Refixation mit PLLA-Implantat } \\
\text { empfohlenes Verfahren }\end{array}$ \\
\hline $\begin{array}{l}\text { Walsh et al. } \\
\text { [26] }\end{array}$ & IV & $\begin{array}{c}8 \\
(12-15 \mathrm{~J})\end{array}$ & $\begin{array}{l}\text { überwiegend } \\
\text { chondral }\end{array}$ & PGA-Biopin & $\begin{array}{l}9(9-11) \\
\text { MR }\end{array}$ & $\begin{array}{lc}\text { IKDC A/B } & 7 x \\
\text { IIKDC C } & 1 x \\
\text { MR } 6 x \text { Knorpelausdünnung }\end{array}$ & $\begin{array}{l}\text { Refixation mit Biopin bei } \\
\text { (osteo)chondraler Läsion empfohlen }\end{array}$ \\
\hline $\begin{array}{l}\text { Nakamura et } \\
\text { al.[111] }\end{array}$ & $\begin{array}{l}\text { case } \\
\text { report }\end{array}$ & $\begin{array}{c}1 \\
(11 \mathrm{~J})\end{array}$ & chondral & PLLA-Biopin & $\begin{array}{c}0,5 \\
(\mathrm{MR}+\mathrm{ASK}+\mathrm{Histologie}) \\
2,8 \\
\text { (MR) } \\
\end{array}$ & $\begin{array}{l}\text { vollständige } \\
\text { Knorpelintegration } \\
\text { (ASK/Histo/MR) }\end{array}$ & $\begin{array}{l}\text { Refixation rein chondrales Fragment } \\
\text { kann bei jungem Patienten zur } \\
\text { kompletten Restitutio führen }\end{array}$ \\
\hline $\begin{array}{l}\text { Uchida et al. } \\
\text { [23] }\end{array}$ & $\begin{array}{l}\text { case } \\
\text { report }\end{array}$ & $\begin{array}{c}3 \\
(11-13 \mathrm{~J})\end{array}$ & chondral & PLLA-Biopin & $\begin{array}{l}2 \\
\text { (MR, } 2 \times \text { second look) }\end{array}$ & $\begin{array}{l}\text { Beschwerdefreiheit } \\
\text { Knorpel stabil eingeheilt }\end{array}$ & $\begin{array}{l}\text { chondrales Fragment an Trochlea kann } \\
\text { erfolgreich bei Jugendlichen refixiert } \\
\text { werden }\end{array}$ \\
\hline
\end{tabular}

Tab. $1 \Delta$ Ergebnisse osteochondraler Refixationen am Knie

\section{Korrespondenzadresse}

\section{Dr. F. Welsch}

Abteilung Sportorthopädie, Knie- und

Schulterchirurgie

Berufsgenossenschaftliche Unfallklinik

Frankfurt am Main, Friedberger Landstraße 430

60389 Frankfurt am Main

Frederic.Welsch@bgu-frankfurt.de

\section{Einhaltung ethischer Richtlinien}

Interessenkonflikt. F. Welsch, T. Stein, P. Minzlaff und R. Hoffmann geben an, dass kein Interessenkonflikt besteht.

Dieser Beitrag beinhaltet keine Studien an Menschen oder Tieren.

The supplement containing this article is not sponsored by industry.

\section{Literatur}

1. Böstman OM, Laitinen OM, Tynninen $O$ et al (2005) Tissue restoration after resorption of polyglycolide and poly-laevo-lactic acid screws. J Bone Joint Surg Br 87(11):1575-1580

2. Brophy RH, Zeltser D, Wright RW et al (2010) Anterior cruciate ligament reconstruction and concomitant articular cartilage injury: incidence and treatment. Arthroscopy 26(1):112-120

3. Buckwalter JA, Mankin HJ (1998) Articular cartilage: degeneration and osteoarthritis, repair, regeneration, and transplantation. Instr Course Lect 47:487-504
4. Chotel F, Knorr G, Simian E et al (2011) Knee osteochondral fractures in skeletally immature patients: French multicenter study. Orthop Traumatol Surg Res 97(8 Suppl):154-159

5. Hoffmann R, Weiler A, Helling HJ et al (1997) Lokale Fremdkörperreaktionen auf biodegradierbare Implantate - eine Klassifikation. Unfallchirurg 100:658-666

6. Hoshino CM, Thomas BM (2010) Late repair of an osteochondral fracture of the patella. Orthopedics 16:270-273

7. Kaplonyi G, Zimmerman I, Frenyo AD et al (1988) The use of fibrin adhesive in the repair of chondral and osteochondral injuries. Injury 19(4):267-272

8. Knapik DM, Harris JD, Pangrazzi G et al (2013) The basic science of continuous passive motion in promoting knee health: a systematic review of studies in a rabbit model. Arthroscopy 29(10):1722-1731

9. Kühle J, Angele P, Balcarek P et al (2013) Treatment of osteochondral fractures of the knee: a metaanalysis of available scientific evidence. Int Orthop (SICOT) 37:2385-2394

10. Mithoefer K, Hambly K, Logerstedt D et al (2012) Current concepts for rehabilitation and return to sport after knee articular repair in the athlete. J Orthop Sports Phys Ther 42(3):254-273

11. Nakamura N, Horibe S, Iwahashi T et al (2004) Healing of a chondral fragment of the knee in an adolescent after internal fixation. A case report. J Bone Joint Surg Am 86-A(12):2741-2746

12. Paar O, Boszotta H (1991) Abscherfrakturen am Knie- und Sprunggelenk. Chirurg 62:121-125

13. Pihlajamäki H, Salminen S, Laitinen $O$ et al (2006) Tissue response to polyglycolide, polydioxanone, polylevolactide, and metallic pins in cancellous bone: an experimental study on rabbits. J Orthop Res 24:1597-1606

14. Plaga BR, Royter RM, Donigian AM et al (1992) Fixation of osteochondral fractures in rabbit knees. A comparison of Kirschner wires, fibrin sealant, and polydioxanone pins. J Bone Joint Surg Br 74(2):292-296
15. Potter HG, Jain SK, Ma Y et al (2012) Cartilage injury after acute, isolated anterior cruciate ligament tear: immediate and longitudinal effect with clinical/MRI follow-up. Am J Sports Med 40(2):276-285

16. Regauer M, Schieker M, Drosse I et al (2010) Volumenzunahme freier artikulärer Knorpel- und Knochen-Knorpel-Fragmente im zeitlichen Verlauf. AGA-Kongress Wien Abstrakt Res 22:68

17. Rehnitz C, Weber MA (2014) Morphologische und funktionelle Knorpeldiagnostik. Radiologe 54:599-617

18. Rosslenbroich S, Raschke M, Petersen W (2012) Akute osteochondrale Läsion nach Patellaluxation. Unfallchirurg 115:392-396

19. Shah N, Meislin R (2013) Current state and use of biological adhesives in orthopedic surgery. Orthopedics 36(12):945-956

20. Stefancin JJ, Parker RD (2007) First-time traumatic patellar dislocation: a systematic review. Clin Orthop Relat Res 455:93-101

21. Törmälä $P$ (1992) Biodegradable self-reinforced composite materials: manufacturing structure and mechanical properties. Clin Mater 10:29-34

22. Touten Y, Adachi N, Deie M et al (2007) Histologic evaluation of osteochondral loose bodies and repaired tissues after fixation. Arthroscopy 23(2):188-196

23. Uchida R, Toritsuka Y, Yoneda K et al (2012) Chondral fragment of the lateral femoral trochlea of the knee in adolescents. Knee 19(5):719-723

24. Vasiliadis HS, Lindahl A, Georgoulis AD et al (2011) Malalignment and cartilage lesions in the patellofemoral joint treated with autologous chondrocyte implantation. Knee Surg Sports Traumatol Arthrosc 19(3):452-457

25. Wachowski MM, Floerkemeier T, Balcarek $P$ et al (2011) Mittelfristige klinische und kernspintomographische Ergebnisse nach Refixation osteochondraler Fragmente mit resorbierbaren Implantaten. Z Orthop Unfall 149:61-67 
26. Walsh SJ, Boyle MJ, Morganti V (2008) Large osteochondral fractures of the lateral femoral condyle in the adolescent: outcome of bioabsorbable pin fixation. J Bone Joint Surg Am 90:1473-1478

27. Wouters DB, Bos RR, van Horn JR et al (2008)

Should in the treatment of of osteochondritis dissecans biodegradable or metallic fixation devices be used? A comparative study in goat knees. J Biomed Mater Res B Appl Biomater 84:154-164

28. Wouters DB, Burgerhof JG, de Hosson JT et al

(2011) Fixation of osteochondral fragments in the human knee using Meniscus Arrows. Knee Surg

Sports Traumatol Arthrosc 19(2):183-188 